\title{
The relationship between work and home characteristics and work engagement in medical residents
}

\author{
Hanne Verweij ${ }^{1}$ (D) $\cdot$ Madelon L. M. van Hooff ${ }^{2}$ Frank M. M. A. van der Heijden ${ }^{3} \cdot$ Jelle T. Prins $^{4}$ • \\ Antoine L. M. Lagro-Janssen ${ }^{5}$ Hiske van Ravesteijn ${ }^{1} \cdot$ Anne E. M. Speckens ${ }^{1}$
}

Published online: 4 July 2017

(C) The Author(s) 2017. This article is an open access publication.

\begin{abstract}
Introduction Work engagement is important for medical residents and the healthcare organizations they work for. However, relatively little is known about the specific predictors of work engagement in medical residents. Therefore, we examined the associations of work and home characteristics, and work-home interference with work engagement in male and female residents.

Methods This study was conducted on a nationwide sample of medical residents. In 2005, all Dutch medical residents $(n=5245)$ received a self-report questionnaire. Path analysis was used to examine the associations between the potential predictors and work engagement.

Results In total, 2115 (41.1\%) residents completed the questionnaire. Job characteristics, home characteristics and work-home interference were associated with work engagement. Important positive contributing factors of work engagement were opportunities for job development, mental demands at work, positive work-home interference and positive home-work interference. Important negative contribut-
\end{abstract}

Hanne Verweij

hanne.verweij@radboudumc.nl

1 Department of Psychiatry, Radboud University Medical Center, Nijmegen, The Netherlands

2 Behavioural Science Institute, Radboud University, Nijmegen, The Netherlands

3 Department of Neuropsychiatry, Vincent van Gogh Institute for Psychiatry, Venlo, The Netherlands

4 MCL Academy, Medical Center Leeuwarden, Leeuwarden, The Netherlands

5 Department of Primary and Community Care, Unit Gender and Womens' Health, Radboud University Medical Center, Nijmegen, The Netherlands ing factors were emotional demands at work and negative home-work interference. The influence of these factors on work engagement was similar in male and female residents. Discussion Opportunities for job development and having challenging work are of high relevance in enhancing work engagement. Furthermore, interventions that teach how to deal skilfully with emotional demands at work and homework interference are expected to be the most effective interventions to enhance work engagement in medical residents.

Keywords Work engagement - Medical residents · Demands \& resources · Work-home interference

\section{What this paper adds}

Work engagement is important for medical residents and the healthcare organizations they work for. However, relatively little is known about the specific predictors in medical residents. Opportunities of job development, mental demands at work and positive work-home interference are important positive contributing factors of work engagement. Emotional demands at work and negative work-home interference are important negative contributing factors of work engagement. In order to enhance work engagement in medical residents it is important to create opportunities for job development and teach residents how to deal with emotional demands at work and home-work interference.

\section{Introduction}

Overall, medical residents are engaged in their work [1]. Work engagement is defined as a positive, fulfilling, work- 
related state of mind that is characterized by vigour (high levels of energy while working), dedication (strongly involved in work), and absorption (fully concentrated and happily engrossed in work) [2]. Work engagement is related to being more committed to the organization, having less turnover intentions, more satisfied clients and better financial outcomes [3, 4]. Within the medical sector work engagement is related to making fewer medical errors. Highly engaged residents reported fewer errors due to inexperience and/or lack of time than those who were less engaged [5]. Although job demands and work pressure are high in hospitals, and stress and burnout are common in medical residents, one-fourth of the residents can still be characterized as highly engaged [1]. Why are some residents more engaged in their work than others? How can we increase work engagement in medical residents? In order to stimulate work engagement in medical residents, we should gain a better understanding of potential contributors of work engagement in this specific population.

According to the Job Demands and Resources theory and previous studies, job resources are positively related to work engagement $[6,7]$. Job resources refer to those aspects of the job that are functional in achieving work goals, reduce job demands and the associated physiological and psychological costs, and/or stimulate personal growth and development [8]. Important resources are opportunities for development, performance feedback, autonomy, skill variety, and social support from colleagues and supervisors. However, the specific resources that predict work engagement may differ per organization [9]. In addition to job resources, job demands also seem to be related to work engagement [10]. Job demands refer to work characteristics that require physical and/or psychological effort and are associated with certain physiological or psychological costs [8]. Although demands are associated with costs and often related to negative work outcomes, job demands that are challenging such as job responsibility, workload, and time urgency might also be positively related to work engagement [11]. However, a study by Mache et al. [10] in 123 surgeons found that job demands were negatively related to work engagement, and that job resources had a greater impact on surgeons' work engagement than job demands. Especially having influence at work, opportunities for development and social support seemed to contribute to work engagement in surgeons. Furthermore, several studies indicate that interaction between demands and resources is relevant as well, suggesting that job resources become more salient and gain their motivational potential when employees are confronted with high job demands [6, 12].

Less is known about the potential influence of home characteristics on work engagement, but several studies indicated that one's functioning at home might impact one's functioning at work and vice versa [13-16]. A study by
Bakker et al. [17] found that home resources and demands were associated with work engagement in a sample of 323 Dutch dual-earner couples, whereas a longitudinal study by Hakanen et al. [18] did not find an effect of home demands or resources on work engagement in Finnish dentists $(n=$ 2555).

The interference between the work and home domain might also be related to work engagement $[19,20]$. This work-home interference and home-work interference can be experienced negatively when demands from the work and family roles are incompatible, such that participation in one role makes it difficult to participate in the other, and positive when positive experiences from one role make it easier to enhance participation in the other role [21, 22]. A study by Montgomery et al. [19] found that positive interference between the home and work domain was correlated with feelings of dedication at work.

With the increasing percentage of women within the medical profession it is very relevant to examine gender differences in work engagement and their contributing factors [23]. Prins et al. [1] indicated that female residents report significantly less vigour than male residents. Furthermore, Verweij et al. [24] found gender differences in contributing factors to burnout. In females, social support from family or partner seemed protective against burnout. In males, social support from colleagues and participation in decisionmaking at work seemed more important. As burnout and work engagement are negatively correlated, we might suspect that there are gender differences in work engagement as well. In addition, Rothbard [25] found that a positive affect towards the home/family life was related to work engagement only among women. This could indicate that the potential contributors of work engagement might differ between male and female residents.

The aim of the present study was to test a model including all the potential contributing factors of work engagement as described before, in order to examine which factors are most important. This will inform us about how to enhance work engagement. In addition, we explored whether there are gender differences with regard to possible contributing or protective factors of work engagement. We formulated the following hypotheses:

Hypothesis 1 We expect (a) the job and home demands to be negatively related to work engagement, (b) the job and home resources to be positively related to work engagement, (c) the job/home resources to be more strongly related to work engagement if job/home demands are high, (d) positive work-home/home-work interference to be positively related to work engagement, and negative workhome/home-work interference to be negatively related to work engagement. 
Hypothesis 2 We expect the model to be different for male and female residents; we expect the home demands and resources to be more strongly related to work engagement in female residents than in male residents.

\section{Methods}

\section{Participants and procedure}

This study was conducted in a large sample of Dutch medical residents. Data were collected in a previous study aimed at gaining insight into the prevalence of burnout and work engagement in medical residents in the Netherlands [1]. All 5245 Dutch medical residents in training on 1 October 2005 were invited to take part in the survey. They received a self-report questionnaire at their home address and they could choose to complete the questionnaire anonymously by hand or online. Participation was voluntary. A cover letter was attached and explained the purpose of the study and emphasized anonymity. All residents were sent three reminders and a non-response form.

At the time of data collection, ethical approval was not required. However, all participants were informed about the study, participation was voluntary and anonymity was guaranteed, also by using a third party to blind the respondents. We ensured that no possible harm could come to the participants of our study.

\section{Measures}

\section{Demographics and occupational characteristics}

Respondents provided information on: gender; age; type of specialty and number of years in training.

\section{Work engagement}

We used the Utrecht Work Engagement Scale to measure work engagement [26]. This questionnaire consists of 15 items measuring three engagement subscales: Vigour (5 items; $\alpha=0.80$ ), dedication (5 items; $\alpha=0.88$ ), and absorption (5 items; $\alpha=0.78$ ). Items were rated on a 7 point Likert scale ranging from 0 'never' to 6 'always'. Example items: 'At my job, I feel bursting with energy.' (vigour); 'My job inspires me.' (dedication); 'When I am working, I forget everything else around me.' (absorption).

\section{Job demands}

Workload (4 items, $\alpha=0.87$ ), emotional demands at work (6 items, $\alpha=0.79$ ) and mental demands at work (4 items, $\alpha=0.77)$ were each measured by scales of the Question- naire on the Experience and Evaluation of Work [27]. Items were rated on a 5-point Likert scale ranging from 1 'never' to 5 'always'. Example items: 'Do you have to work very fast?' (workload); 'Is your work emotionally demanding?' (emotional demands), and 'Does your work demand a lot of concentration?' (cognitive demands).

\section{Job resources}

Six job resources were measured using scales of the Questionnaire on the Experience and Evaluation of Work [27]: Job autonomy ( 3 items, $\alpha=0.73$ ), job development ( 3 items, $\alpha=0.80$ ), social support from colleagues (3 items, $\alpha=$ $0.84)$, performance feedback (5 items, $\alpha=0.83$ ), supervisory coaching ( 6 items, $\alpha=0.86$ ), and participation in decision making ( 4 items, $\alpha=0.77$ ). All items were scored on a 5-point rating scale ranging from 1 ('never/ poor/totally disagree') to 5 ('always/good/totally agree'). Example items: 'Do you have freedom in carrying out your work activities?' (autonomy); 'At work I am given the opportunity to develop my personal strengths' (job development); 'Can you, when necessary, ask your colleagues for help?' (social support from colleagues); 'I receive enough feedback from my supervisor in regards to my work' (performance feedback); 'My supervisor uses his/her influence to help me solve my problems at work' (supervisory coaching); and 'I feel that I am involved in making important decisions' (participation in decision making).

\section{Home demands}

Three home demands were measured using scales used in previous studies [19, 28]: Homeload (5 items, $\alpha=0.75$ ), emotional demands ( 3 items, $\alpha=0.76$ ) and mental demands ( 3 items, $\alpha=0.88$ ). All items were scored on a 5point rating scale ranging from 1 ('never') to 5 ('always'). Example items: 'Do you have to carry out a lot of tasks at home [household/caring tasks]?' (homeload); 'Are you confronted with situations in your private life that are emotionally charged?' (emotional demands); and 'Do you have to plan and organize a lot of things in relation to your home life?' (mental demands).

\section{Home resources}

Three home resources were measured using scales used in previous studies [15, 17]: personal autonomy (4 items, $\alpha=0.82$ ), social support from partner/family (4 items, $\alpha=$ 0.87 ), and opportunity for personal development (3 items, $\alpha=0.88$ ). All items were scored on a 5-point rating scale ranging from 1 ('never') to 5 ('always'). Example items: 'I manage daily life at home' (personal autonomy); 'My family/partner pays attention to my feelings and problems' 
(social support from partner/family); and 'I can develop my talents during my free time' (opportunity for personal development).

\section{Work-home interference}

The Survey Work-home Interaction NijmeGen (SWING) was used to measure work-home interference [29]. This questionnaire measures four subscales: positive work-home interference (3 items, $\alpha=0.42$ ), negative work-home interference ( 3 items, $\alpha=0.73$ ), positive home-work interference (3 items, $\alpha=0.68$ ), and negative home-work interference (3 items, $\alpha=0.79$ ). All items were scored on a 5-point rating scale ranging from 1 ('never') to 5 ('always'). Example items: 'How often does it happen that after a pleasant working day, you feel more in the mood to engage in activities with your spouse/family/friends?' (positive work-home interference); 'How often do you find it difficult to fulfil your domestic obligations because you are constantly thinking about your work?' (negative work-home interference); 'How often does it happen that after spending a pleasant weekend with your spouse/family/friends, you have more fun in your job?' (positive home-work interference); and 'How often do you not fully enjoy your work because you worry about your home situation?' (negative home-work interference).

\section{Data analysis}

We analyzed the data by means of path analysis, using the Mplus7 statistical software package [30]. Path analysis is a subset of structural equation modelling using only measured variables and no latent variables. We developed a model and examined the associations between the specific work and home demands and resources and the four types of work-home interference on the one hand, and the three work engagement subscales on the other hand. These associations or pathways in the models represent hypotheses, which are based on previous research and theoretical propositions as described in the introduction. We included work characteristics in our analyses first, and added home characteristics in a next step, in order to be able to examine if including home characteristics explained an additional proportion of the variance in work engagement, beyond the effects of work characteristics. To examine possible gender differences in these associations we employed multi-group path analysis in Mplus7 [30]. The improvement in fit of the models was assessed using the chi-square difference test, the root mean square error of approximation (RMSEA) and the comparative fit index (CFI). Values of 0.90 and higher (CFI) and 0.08 or lower (RMSEA) indicate an acceptable fit [31]. Because of the large dataset and the number of vari- ables included in this model we chose to indicate $p$-values below 0.01 as significant in order to decrease the chance of type 1 errors. Standardized path coefficients (Beta values) were calculated to determine the possible predicting factors of work engagement. These indicate the patterns of associations between the predicting factors and work engagement. Standardized path coefficients $(\beta)$ with values of less than 0.10 can be interpreted as small effects, values of around 0.30 can be interpreted as medium effects and values above 0.50 can be interpreted as large effects [32].

Before testing our hypotheses, the items representing the constructs in the research model were subjected to confirmatory factor analyses using Mplus7. All measures we used were employed in previous studies and generally proved to be valid. The fit indices of the measurement model were near adequate, $\chi^{2}(3444)=17,382.40, \mathrm{RMSEA}=0.04, \mathrm{CFI}=$ 0.85 , standardized root mean residual $=0.05$. All items loaded significantly on the predicted factor. However, one item from the homeload construct had a low factor loading $(<0.40)$. We decided not to remove this item because of its conceptual importance and because the internal consistency of the construct, expressed with Cronbach's $\alpha$ coefficient, was considered sufficient $(\alpha=0.75)$.

\section{Results}

\section{Study population}

Of the 5245 residents who were invited, 105 indicated that they were no longer residents. Of the remaining 5140 residents, $125(2.4 \%)$ indicated that they did not wish to participate. In total, $2115(41.1 \%)$ completed the questionnaire [1]. Characteristics of the respondents are presented in Table 1 .

\section{General model for work engagement}

Table 2 provides an overview of the fit indices for the models that were estimated. In the first model (M1), only job demands and job resources were modelled to be related to engagement. As indicated by the CFI and RMSEA value, this model fitted well with the data. Building on this first model, we estimated a second model (M2) that was identical to M1, except that it additionally included the associations between home demands and home resources and engagement. As can be seen in Table 2, adding these paths significantly improved the fit of our model. In Model 3 (M3), we extended M2 by including the interactions between job demands and job resources, in order to examine the proposition of the Job Demands and Resources model that demands moderate the association between resources and engagement [33]. In order to avoid problems of multi- 
Table 1 Characteristics of the respondents $(n=2115)$

\begin{tabular}{llll}
\hline Variable & Mean (SD) & $N$ & $\%$ \\
\hline Gender & & & \\
Female & & 1290 & 61.0 \\
Male & 820 & 38.8 \\
Missing & 5 & 0.2 \\
Age, range 23-58 years & $31.5(3.5)$ & & \\
Years in training & $3.0(1.5)$ & & \\
Medical specialty in groups & & 2115 & \\
(Internal) medical specialties & & 951 & 45.0 \\
Surgical specialties & & 649 & 30.7 \\
Supportive/diagnostic specialties & & 270 & 12.8 \\
Psychiatry & & 242 & 11.4 \\
Missing & & 3 & 0.1 \\
\hline
\end{tabular}

$S D$ standard deviation

collinearity, these interactions were computed using meancentred scores [34]. As M3 did not provide a better fit than M2, these interactions were omitted from further analyses. Model 4 (M4) was therefore also based on M2, and included the interactions between home demand and home resources. Again, this model did not improve model fit compared with M2. Therefore, our final model (M5), in which the paths between positive and negative home-work and work-home interference and engagement were estimated, was also based on M2. This model not only fitted significantly better than M2, but also showed a very good fit in an absolute sense.

\section{Gender differences in the work engagement model}

Results of the gender-specific analyses are presented in Table 2. We started by modelling the paths that were included in M5 in female and male residents separately. Initially, we imposed equality constraints on all structural paths, thus assuming the strength of all associations to be similar for both sexes. This model provided a good fit to the data (M6). Subsequently, in a second model (M7) we removed these equality constraints to examine if this resulted in a better fitting model. Table 2 shows that M7 (without constraints) did not fit better than M6 (with constraints). Therefore, our data do not provide evidence for gender differences in the associations between work and home characteristics and work-home interference, and engagement.

\section{Contributing and protective factors of engagement}

Fig. 1, 2 and 3 provide the graphical representation of the final model (M5). In order to reduce the complexity of the figure, we divided the figure into three separate figures, each representing one subscale of work engagement. Furthermore, the figures only show the significant associations and standardized path coefficients (Beta values). The factors in this model explain a significant proportion of variance of vigour $\left(\mathrm{R}^{2}=0.305\right)$, dedication $\left(\mathrm{R}^{2}=0.358\right)$ and absorption $\left(\mathrm{R}^{2}=0.206\right)$. All significant associations of the three subscales of work engagement are described below.

\section{Vigour}

With regard to the positive associations with vigour, we found that mental demands at work, social support from colleagues, job development, supervisory coaching, mental demands at home, positive work-home interference and positive home-work interference were positively associated with vigour. Emotional demands at work, negative workhome interference and negative home-work interference were negatively associated with vigour.

\section{Dedication}

Mental demands at work, job development, social support from colleagues, positive work-home interference and pos-

Table 2 Fit indices for different models

\begin{tabular}{|c|c|c|c|c|}
\hline & $\chi^{2}(\mathrm{df})$ & $\Delta \chi^{2}(\mathrm{df})$ & RMSEA & CFI \\
\hline $\begin{array}{l}\text { M1: work demands and work resources as predictors of work } \\
\text { engagement }\end{array}$ & $438.85(111)$ & & 0.04 & 0.93 \\
\hline $\begin{array}{l}\text { M2: M1 + home demands and home resources as predictors of } \\
\text { work engagement }\end{array}$ & $360.22(93)$ & $78.63(18)^{\mathrm{a}}$ & 0.04 & 0.94 \\
\hline $\begin{array}{l}\text { M3: M2 }+ \text { interactions work demands } \times \text { work resources as predic- } \\
\text { tors of work engagement }\end{array}$ & $317.11(39)$ & $43.11(54)$ & 0.06 & 0.94 \\
\hline $\begin{array}{l}\text { M4: M } 2+\text { interactions home demands } \times \text { home resources as pre- } \\
\text { dictors of work engagement }\end{array}$ & $326.44(6)$ & $33.78(27)$ & 0.05 & 0.94 \\
\hline $\begin{array}{l}\text { M5: M2 + WHIpos, WHIneg, HWIpos, and HWIneg as predic- } \\
\text { tors of work engagement }\end{array}$ & $82.39(81)$ & $277.83(12)^{\mathrm{a}}$ & 0.00 & 1.00 \\
\hline M6: M5 + Gender. Equality constraints on all structural paths & $249.91(219)$ & & 0.01 & 0.99 \\
\hline M7: M5 + Gender. No constraints for paths & $200.65(162)$ & $49.26(57)$ & 0.02 & 0.99 \\
\hline
\end{tabular}

WHI work-home interference, $H W I$ home-work interference, RMSEA root mean square error of approximation, $C F I$ comparative fit index

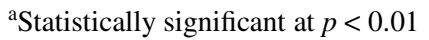


Fig. 1 Path analysis of vigour. Only associations that were statistically significant at $p<0.01$ are presented. (WHI work-home interference, $H W I$ home-work interference)

Fig. 2 Path analysis of dedication. Only associations that were statistically significant at $p<0.01$ are presented. (WHI work-home interference, $H W I$ home-work interference)

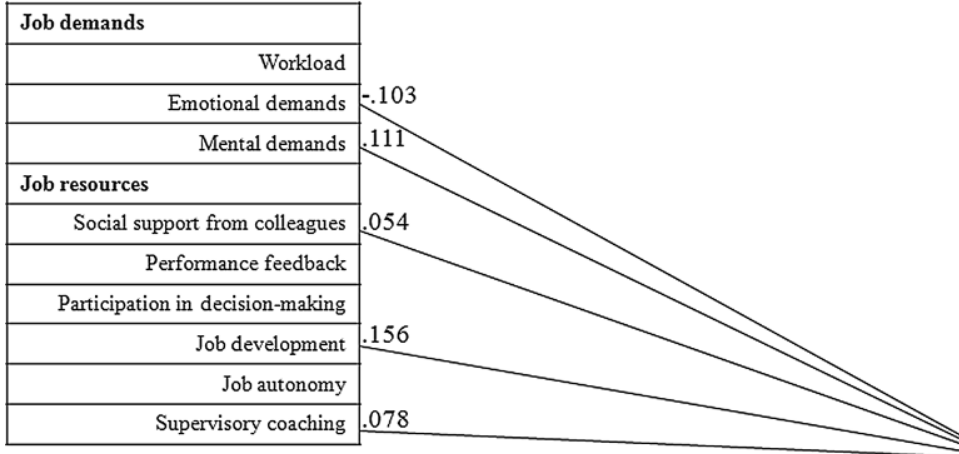

\begin{tabular}{|r|}
\hline \multicolumn{1}{|l|}{ Home demands } \\
\hline \multicolumn{2}{|c|}{ Home workload } \\
\hline Mentional demands demands \\
\hline Home resources \\
\hline Social support from partner/family \\
\hline Personal autonomy \\
\hline Personal development \\
\hline
\end{tabular}

Work-Home interaction

WHI positive

WHI negative

HWI positive

HWI negative

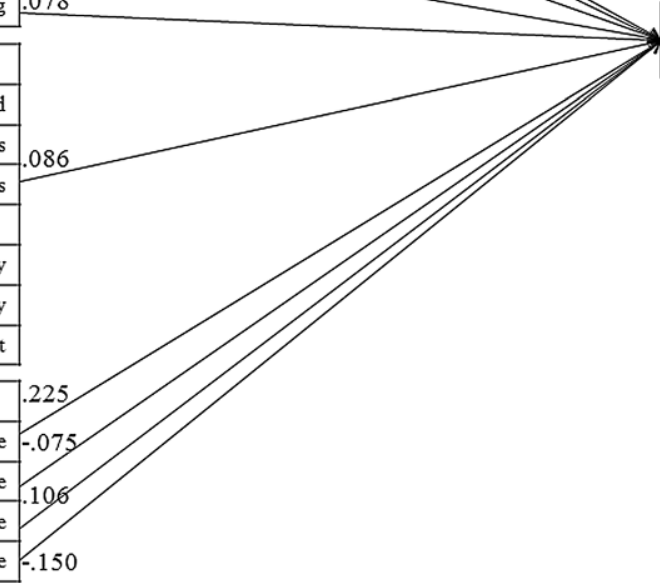

Vigour

$\mathrm{R}^{2}=.305$

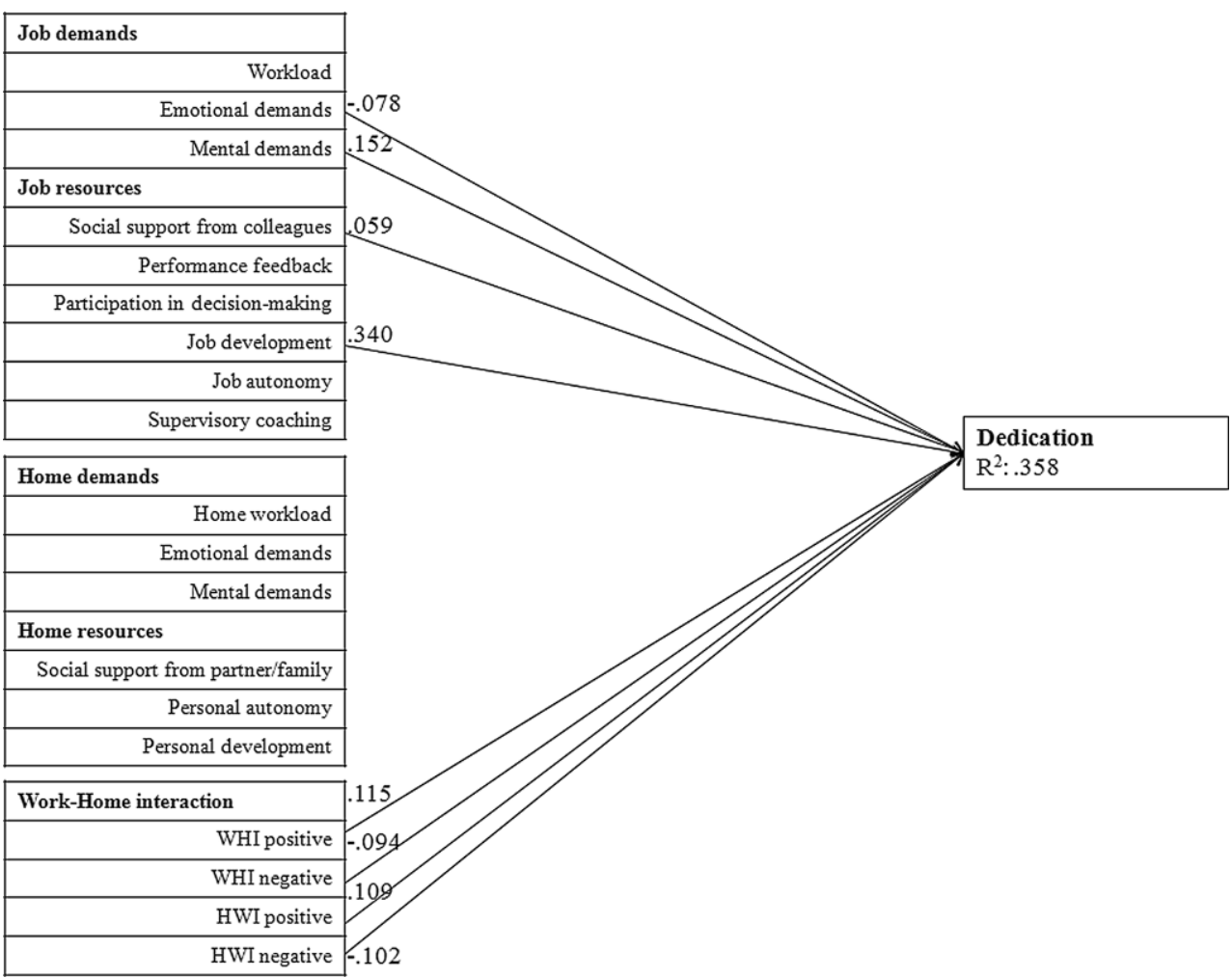


Fig. 3 Path analysis of absorption. Only associations that were statistically significant at $p<0.01$ are presented. (WHI work-home interference, $H W I$ home-work interference)

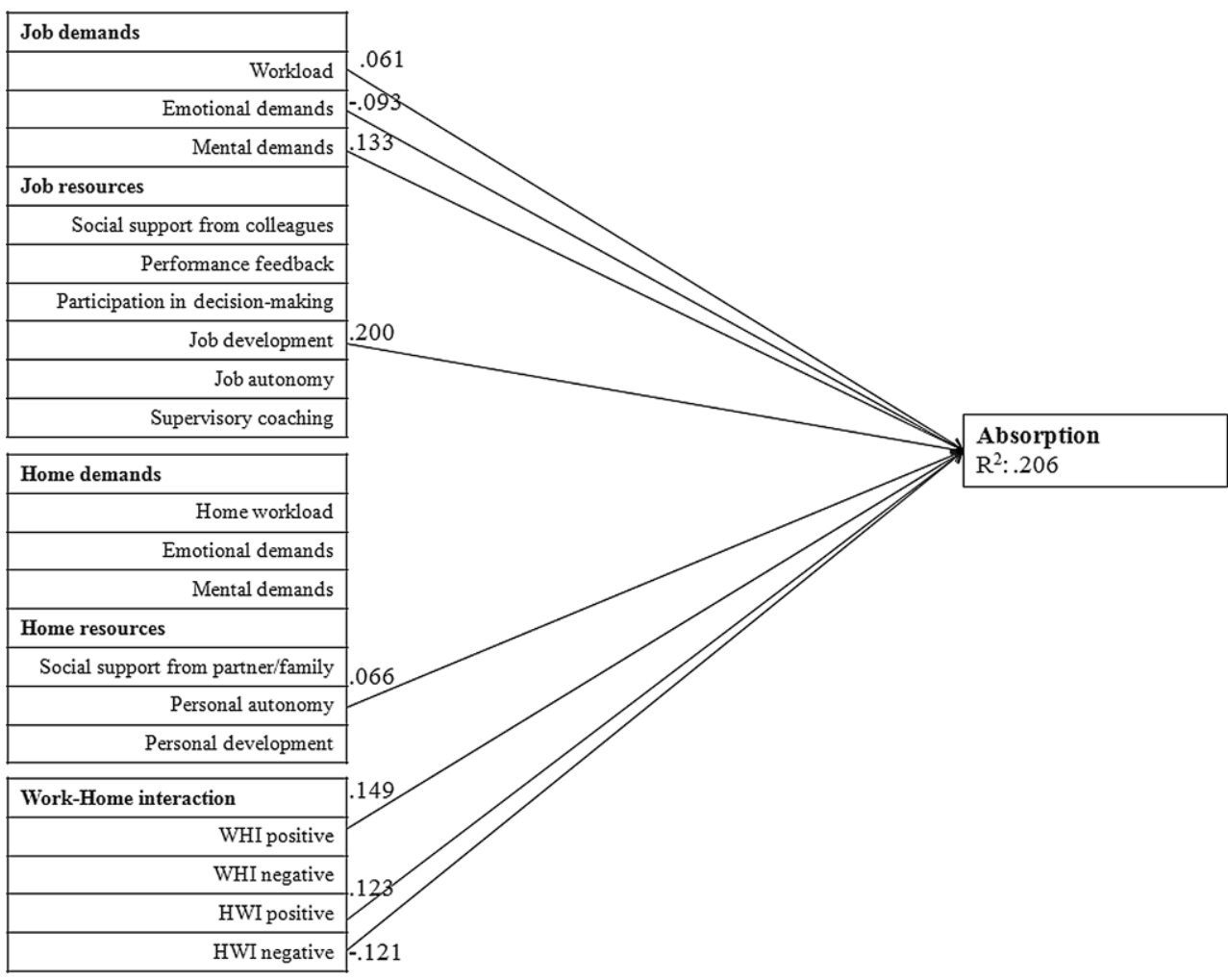

itive home-work interference were positively associated with dedication. Emotional demands at work, negative work-home interference and negative home-work interference were negatively associated with dedication.

\section{Absorption}

Mental demands at work, job workload, job development, personal autonomy, positive work-home interference and positive home-work interference were positively related to absorption. Emotional demands at work and negative homework interference were negatively associated with absorption.

\section{Discussion}

The aim of this study was to examine the associations of work and home characteristics and work-home interference with work engagement in Dutch male and female medical residents.

The results partly confirmed our hypothesized model in that we found work demands and resources, home demands and resources, and work-home and home-work interference to be associated with work engagement. However, we did not find support for hypothesis 1c; demands did not moderate the association between resources and engagement. Furthermore, our data do not provide any evidence for pos- sible gender differences in the associations between work and home characteristics and work-home interference, and engagement (hypothesis 2).

Examining these associations in more detail, we see that especially opportunities for job development, positive work-home interference, positive home-work interference and mental demands at work were important contributing factors to all three subscales of work engagement. Interestingly, 'mental demands at work' was positively rather than negatively related to work engagement, suggesting that mental demands are considered to be challenging rather than exhausting [11]. Emotional demands at work and negative home-work interference had an important negative impact on work engagement.

There was only a very small effect of home demands and resources on work engagement. The work-home interferences, both the negative and positive home to work interference and work to home interference, seemed more important for experiencing work engagement. This might indicate that for medical residents the home situation in itself is not that influential, but the interference between the home and work domain has a greater impact on engagement at work.

Furthermore, we did not find gender differences regarding the associations in the model. This is in contrast with our expectations, but it is in line with research in a heterogeneous occupational sample $(n=846)$ by Korunka et al. [35], who did not find gender differences either in the rela- 
tionship between job resources and work engagement. The strengths of the associations between resources, demands and work-home interferences with work engagement in our research are similar for male and female residents. However, previous research in medical residents demonstrated that female residents experienced more home demands and more negative home-to-work interference, while male residents reported more social support from colleagues and supervisory coaching [24]. The circumstances seemed slightly better for male residents as they reported more of what is needed for being engaged, which might explain that male residents also reported more vigour compared with female residents [1].

Although, more insight into the specific contributing factors of work engagement might lead to a better functioning of the residents themselves, the organization and a higher quality of patient care, it is also important to be aware of the possible downsides of work engagement [36]. Bakker et al. [37] suggested that there might be a limit to engagement. Overly engaged workers may often work overtime, forget to rest or maintain their personal relationships and hence experience more work-family conflict. In a medical culture with its emphasis on commitment, the high responsibility in patient care and expectation to work overtime, this is important to bear in mind.

\section{Strengths and limitations}

One of the strengths of this study is the large and representative population. All medical residents in the Netherlands were invited to participate, which resulted in a large sample with residents from different regions of the country, different hospital settings and different medical specialties.

As any study, the present study also has limitations. First of all, the data were collected at the end of 2005. So, our findings might have been overhauled by social or cultural changes that have since taken place or by developments in the postgraduate training of medical residents. However, we believe that although in 10 years the absolute values of work engagement and its contributors might have slightly changed, the relationships between these variables and work engagement have not been affected by such changes. The Job Demands-Resources model, on which we based our hypotheses, assumes that the availability of job resources helps in dealing with job demands and plays a motivational role, which fosters work engagement [38]. There is no reason to believe that this process or the specific characteristics associated with work engagement in medical residents have changed during the past decade because the nature of the job has not essentially changed.

Secondly, the data were collected at one point in time, so the associations cannot be interpreted causally. For instance, it could also be that medical residents with high levels of engagement more easily identify positive work-home interference. However, longitudinal research by Hakanen et al. [18] did not find evidence for the reversed effect of work engagement on job demands and resources.

Thirdly, although considering the nature of the survey the response rate was quite high $(41 \%)$, more than half of the invited medical residents did not respond. This could have led to a selection bias. Common reasons for not responding in those who took the trouble to send in their non-response form appeared to be a lack of time (22\%) and a lack of energy (11\%). This might have resulted in an overestimation of work engagement in medical residents. However, again, this might especially have affected the absolute levels of burnout and its contributors instead of their relationships. Furthermore, as all measures were based on self-reports, common method variance may have biased our findings. Ideally, future research should combine self-report questionnaires with more objective measures, such as computerize tasks, observations and organisation's records.

\section{Future research and practical implications}

Future research should use longitudinal designs to better explore the predictive nature of the associations found in our model and the possible long-term consequences of work engagement in relationship with health. Furthermore, forthcoming studies about work engagement would benefit from a focus on interventions, and study the impact and effectiveness of these interventions.

With regard to implications for clinical practice, our findings support the improvement of resources at work, such as better opportunities for career development, personal development and creating a supportive culture. Furthermore, residents should learn how to deal with emotional demands at work, for example by facilitating regular supervision or peer supervision where junior doctors can exchange difficult situations they might have encountered. Alternatively, one might stimulate curricular initiatives that incorporate aspects of allowing and regulating emotions, such as narrative medicine or mindfulness training $[39,40]$. In addition to changing circumstances at work, there should be more attention to the benefits of the decreasing negative interference and stimulating positive interference between home and work. Hospitals could provide facilities supporting the work-life balance such as flexible working hours, self-scheduling, part-time work, and childcare. Secondly, and this might even be more important, hospitals should create a culture in which residents feel supported in using these facilities without experiencing a negative effect on their career opportunities [41]. 
Acknowledgements This study has been unrestrictedly supported by 'Stichting SWG', 'CarriereCentrum Zorg' (Utrecht, the Netherlands) and Springer Media (Houten, the Netherlands). They supported the initial research project in 2005 by unrestricted grants; this did not influence the design and execution of the research project. No funding was granted for this additional study.

\section{Compliance with ethical guidelines}

Conflict of interest H. Verweij, M. van Hooff, F.M.M.A. van der Heijden, J.T. Prins, A. Lagro-Janssen, H. van Ravesteijn and A. Speckens declare that they have no competing interests.

Ethical standards At the time of data collection, ethical approval was not required. However, all participants were informed about the study, participation was voluntary and anonymity was guaranteed, also by using a third party to blind the respondents. We ensured that no possible harm could come to the participants of our study.

Open Access This article is distributed under the terms of the Creative Commons Attribution 4.0 International License (http:// creativecommons.org/licenses/by/4.0/), which permits unrestricted use, distribution, and reproduction in any medium, provided you give appropriate credit to the original author(s) and the source, provide a link to the Creative Commons license, and indicate if changes were made.

\section{References}

1. Prins JT, Hoekstra-Weebers JEHM, Gazendam-Donofrio SM, et al. Burnout and engagement among resident doctors in the Netherlands: a national study. Med Educ. 2010;44:236-47.

2. Schaufeli WB, Bakker AB. Job demands, job resources, and their relationship with burnout and engagement: a multi-sample study. J Organ Behav. 2004;25:293-315.

3. Bakker AB, Schaufeli WB, Leiter MP, Taris TW. Work engagement: an emerging concept in occupational health psychology. Work Stress. 2008;22:187-200.

4. Simpson MR. Engagement at work: a review of the literature. Int J Nurs Stud. 2009;46:1012-24.

5. Prins JT, Van der Heijden FMMA, Hoekstra-Weebers JEHM, et al. Burnout, engagement and resident physicians' self-reported errors. Psychol Health Med. 2009;14:654-66.

6. Bakker AB, Demerouti E. Towards a model of work engagement. Career Dev Int. 2008;13:209-23.

7. Hakanen JJ, Bakker AB, Schaufeli WB. Burnout and work engagement among teachers. J School Psychol. 2006;43:495-513.

8. Bakker AB, Demerouti E, Verbeke W. Using the job demands-resources model to predict burnout and performance. Hum Resour Manage. 2004;43:83-104.

9. Schaufeli WB. Work engagement: what do we know and where do we go. Rom J Appl Psychol. 2012;14:3-10.

10. Mache S, Vitzthum K, Klapp BF, Danzer G. Surgeons' work engagement: influencing factors and relations to job and life satisfaction. Surgeon. 2014;12:181-90.

11. Crawford ER, LePine JA, Rich BL. Linking job demands and resources to employee engagement and burnout: a theoretical extension and meta-analytic test. J Appl Psychol. 2010;95:834-48.

12. Hakanen JJ, Bakker AB, Demerouti E. How dentists cope with their job demands and stay engaged: the moderating role of job resources. Eur J Oral Sci. 2005;113:479-87.

13. Bakker AB, Geurts SAE. Toward a dual-process model of workhome interference. Work Occup. 2004;31:345-66.
14. Byron K. A meta-analytic review of work-family conflict and its antecedents. J Vocat Behav. 2005;67:169-98.

15. Demerouti E, Bakker AB, Voydanoff P. Does home life interfere with or facilitate job performance? Eur J Work Organ Psy. 2010;19:128-49.

16. Bakker AB, ten Brummelhuis LL, Prins JT, van der Heijden FMMA. Applying the job demands-resources model to the workhome interface: a study among medical residents and their partners. J Vocat Behav. 2011;79:170-80.

17. Bakker AB, Demerouti E, Schaufeli WB. The crossover of burnout and work engagement among working couples. Hum Relat. 2005;58:661-89.

18. Hakanen JJ, Schaufeli WB, Ahola K. The Job Demands-Resources model: a three-year cross-lagged study of burnout, depression, commitment, and work engagement. Work Stress. 2008;22:224-41.

19. Montgomery AJ, Peeters MCW, Schaufeli WB, Den Ouden M. Work-home interference among newspaper managers: its relationship with burnout and engagement. Anxiety Stress Coping. 2003;16:195-211.

20. Mostert K, Peeters M, Rost I. Work-home interference and the relationship with job characteristics and well-being: a South African study among employees in the construction industry. Stress Health. 2011;27:e238-e51.

21. Grzywacz JG, Marks NF. Reconceptualizing the work-family interface: an ecological perspective on the correlates of positive and negative spillover between work and family. J Occup Health Psychol. 2000;5:111.

22. Greenhaus JH, Beutell NJ. Sources of conflict between work and family roles. Acad Manage Rev. 1985;10:76-88.

23. Velden L, Hingstman L, Heiligers P, Hansen J. Toenemend percentage vrouwen in de geneeskunde: verleden, heden en toekomst. Ned Tijdschr Geneeskd. 2008;152:2165.

24. Verweij H, van der Heijden FM, van Hooff ML, et al. The contribution of work characteristics, home characteristics and gender to burnout in medical residents. Adv Health Sci Educ Theory Pract. 2016; . doi:10.1007/s10459-016-9710-9.

25. Rothbard NP. Enriching or depleting? The dynamics of engagement in work and family roles. Admin Sci Quart. 2001;46:655-84.

26. Schaufeli W, Bakker A. Utrecht work engagement scale: Preliminary manual. Utrecht: Occupational Health Psychology Unit, Utrecht University; 2003.

27. Veldhoven MJPM, Meijman TF. Het meten van psychosociale arbeidsbelasting met een vragenlijst: de vragenlijst beleving en beoordeling van de arbeid (VBBA). Amsterdam: Nederlands Instituut voor Arbeidsomstandigheden NIA; 1994.

28. Peeters MC, Montgomery AJ, Bakker AB, Schaufeli WB. Balancing work and home: how job and home demands are related to burnout. Int J Stress Manag. 2005;12:43.

29. Geurts SAE, Taris TW, Kompier MAJ, Dikkers JSE, Van Hooff MLM, Kinnunen UM. Work-home interaction from a work psychological perspective: development and validation of a new questionnaire, the SWING. Work Stress. 2005;19:319-39.

30. Muthén LK, Muthén BO. Mplus user's guide, 7th ed. Los Angeles: Muthén \& Muthén; 2012.

31. Byrne BM. Structural equation modeling with AMOS: basic concepts, applications, and programming. London: Routledge; 2013.

32. Kline RB. Principles and practice of Structural Equations Modeling. New York: Wiley; 1998.

33. Bakker AB, Demerouti E. The job demands-resources model: state of the art. J Manag Psychol. 2007;22:309-28.

34. Aiken LS, West SG, Reno RR. Multiple regression: testing and interpreting interactions. Newbury Park: SAGE; 1991.

35. Korunka C, Kubicek B, Schaufeli WB, Hoonakker P. Work engagement and burnout: testing the robustness of the Job Demands-Resources model. J Posit Psychol. 2009;4:243-55. 
36. Schaufeli WB, Bakker AB, van der Heijden FMMA, Prins JT. Workaholism, burnout and well-being among junior doctors: the mediating role of role conflict. Work Stress. 2009;23:155-72.

37. Bakker AB, Albrecht SL, Leiter MP. Key questions regarding work engagement. Eur J Work Organ Psychol. 2011;20:4-28.

38. Demerouti E, Bakker AB, Nachreiner F, Schaufeli WB. The job demands-resources model of burnout. J Appl Psychol. 2001;86:499512.

39. Regehr C, Glancy D, Pitts A, LeBlanc VR. Interventions to reduce the consequences of stress in physicians a review and meta-analysis. J Nerv Ment Dis. 2014;202:353-9.

40. Shapiro J. Perspective: does medical education promote professional alexithymia? A call for attending to the emotions of patients and self in medical training. Acad Med. 2011;86:326-32.

41. Dikkers JSE, Geurts SAE, Dulk LD, Peper B, Taris TW, Kompier MAJ. Dimensions of work-home culture and their relations with the use of work-home arrangements and work-home interaction. Work Stress. 2007;21:155-72.

Hanne Verweij MSc, is a researcher at Radboud University Medical Center, Nijmegen, the Netherlands.
Madelon L. M. van Hooff $\mathrm{PhD}$, is assistant professor at the Behavioural Science Institute, Radboud University, Nijmegen, the Netherlands.

Frank M. M. A. van der Heijden $\mathrm{MD}, \mathrm{PhD}$, is psychiatrist at Vincent van Gogh Institute for Psychiatry, Venlo, the Netherlands.

Jelle T. Prins PhD, is dean at MCL Academy of Leeuwarden, the Netherlands and researcher at the Dutch Doctors' Association, the Netherlands.

Antoine L. M. Lagro-Janssen $\mathrm{MD}, \mathrm{PhD}$, is a general practitioner and professor in Women's Studies Medicine at Radboud University Medical Center, Nijmegen, the Netherlands.

Hiske van Ravesteijn $\mathrm{MD}, \mathrm{PhD}$, is a psychiatrist and senior researcher at Radboud University Medical Center, Nijmegen, the Netherlands.

Anne E. M. Speckens MD, $\mathrm{PhD}$, is professor in Psychiatry at Radboud University Medical Center, Nijmegen, the Netherlands. 